\title{
Prospective association between use of electronic cigarettes and use of conventional cigarettes: a systematic review and meta-analysis
}

\author{
Louise Adermark $\mathbb{1}^{1,2}$, Maria Rosaria Galanti ${ }^{3,4}$, Charlotta Ryk ${ }^{5}$, Hans Gilljam ${ }^{3}$ and Linnea Hedman ${ }^{6,7}$
}

${ }^{1}$ Dept of Psychiatry and Neurochemistry, Sahlgrenska Academy, University of Gothenburg, Gothenburg, Sweden. ${ }^{2}$ Dept of Pharmacology, Sahlgrenska Academy, University of Gothenburg, Gothenburg, Sweden. ${ }^{3}$ Dept of Global Public Health, Karolinska Institutet, Stockholm, Sweden. ${ }^{4}$ Centre of Epidemiology and Community Medicine, Stockholm, Sweden. ${ }^{5}$ Swedish Agency for Health Technology Assessment and Assessment of Social Services (SBU), Stockholm, Sweden. ${ }^{6}$ Dept of Public Health and Clinical Medicine, Division Sustainable health, The OLIN Unit, Umeå University, Umeå, Sweden. ${ }^{7}$ Dept of Health Sciences, Division of Nursing, Luleå University of Technology, Luleå, Sweden.

Corresponding author: Louise Adermark (louise.adermark@gu.se)

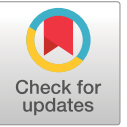

This version is distributed under the terms of the Creative Commons Attribution NonCommercial Licence 4.0. For commercial reproduction rights and permissions contact permissions@ersnet.org

This article has supplementary material available from openres.ersjournals.com

Received: 2 Jan 2021 Accepted: 5 May 2021

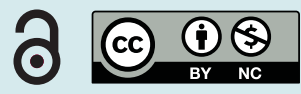

Shareable abstract (@ERSpublications)

This meta-analysis shows that e-cigarette use increases the risk of future initiation or recurrence of cigarette smoking, even after adjusting for potential confounders, suggesting that e-cigarette use could be considered a predictor of subsequent smoking https://bit.ly/3buMccm

Cite this article as: Adermark L, Galanti MR, Ryk C, et al. Prospective association between use of electronic cigarettes and use of conventional cigarettes: a systematic review and meta-analysis. ERJ Open Res 2021; 7: 00976-2020 [DOI: 10.1183/23120541.00976-2020].

\section{Abstract}

Objective The aim of this systematic review and meta-analysis was to assess the association between e-cigarette use and subsequent initiation or recurrence of cigarette smoking.

Data sources A systematic literature search was finalised on 11 November 2019 using PubMed (including MEDLINE), EMBASE, Cochrane Library, Scopus, PubMed Health, NICE Evidence Search, PROSPERO, CRD and PsycInfo.

Study selection Studies were included if meeting the following criteria: reporting empirical results; longitudinal observational design with a minimum of 3 months of follow-up; including general population samples; allowing for the comparison between users and nonusers of e-cigarettes. Studies rated as having high risk of bias were excluded. Studies were independently assessed by at least two authors. The procedures described by PRISMA were followed, and the quality of evidence was rated using GRADE.

Data synthesis 30 longitudinal studies from 22 different cohorts assessing e-cigarette use among nonsmokers or never-smokers at baseline, and subsequent use of cigarette smoking at follow-up, were included in this review. A random-effects meta-analysis based on 89076 participants showed a pooled unadjusted odds ratio (OR) of cigarette smoking among baseline nonsmoker e-cigarette users compared with nonusers of 4.68 (CI 3.64-6.02), while the adjusted OR was 3.37 (CI 2.68-4.24). These results were consistent irrespective of whether the outcome was measured as ever-smoking or as past 30-day smoking. The evidence was graded as moderate.

Conclusions Use of e-cigarettes may predict the initiation or recurrence of cigarette smoking.

\section{Introduction}

Since their introduction in 2003, the awareness and use of electronic cigarettes (e-cigarettes) have steadily increased [1, 2]. Especially, the e-cigarette has gained popularity among adolescents, with rapidly rising prevalence of past 30-day use. In the USA, over $25 \%$ of high school students report current use [3, 4], and the prevalence of e-cigarette use now reaches similar or even higher levels than regular smoking [5, 6].

E-cigarette use is more prevalent among cigarette smokers compared to nonsmokers [6, 7]. In theory, substituting regular smoking with e-cigarettes could lead to both individual and public health beneficial effects. However, recent studies suggest that e-cigarette use is increasing especially among nonsmokers and experimental smokers [8]. Furthermore, several longitudinal studies have reported that e-cigarette use is 
associated with an increased risk of cigarette smoking initiation [9-11]. In fact, studies suggest that even youths at low risk of becoming conventional smokers find e-cigarettes appealing [10, 12-14]. Therefore, it is possible that initiation of e-cigarette use may promote the subsequent initiation of cigarette smoking, thus impairing further decreases in population smoking prevalence. While data from Canada seems to support an increase in smoking prevalence [8], similar trends have not yet been reported in the USA or England [6, 8, 15].

The increased risk for smoking initiation among nonsmoking e-cigarette users has been highlighted in several reviews [16-19]. However, the number of publications addressing this issue is rapidly growing. Furthermore, some of these reviews include articles with study designs that are subject to numerous limitations and/or studies with a cross-sectional design. As the accuracy of prevalence estimates derived from nonprobability-based samples warrants consideration, we thus conducted a systematic review and meta-analysis of longitudinal studies with a low or moderate risk of bias that assessed the transition from nonsmoking to cigarette smoking among e-cigarette users and nonusers. This review is based on data previously published as a report from The Swedish Agency for Health Technology Assessment and Assessment of Social Services (SBU) (report 312, SBU 2020/431).

\section{Methods}

\section{Research question}

The present review focused on the following research question: Is e-cigarette use among nonsmokers associated with an increased risk for subsequent smoking? The PICO (Population, Intervention, Comparison, and Outcome) schema used to establish the eligibility criteria is presented in table 1.

\section{Literature search}

A comprehensive literature search of PubMed (including MEDLINE), EMBASE, Cochrane Library, Scopus, PubMed Health, NICE Evidence Search, PROSPERO, CRD (DARE) and PsycInfo was conducted in December 2018 and updated on 11 November 2019. Search terms were based on the following keywords: electronic cigarettes, vaping, vaporised nicotine, electronic nicotine delivery system; combined with cigarettes, smoking, combustible tobacco. Details of the literature search can be found in table S1.

\section{Inclusion and exclusion criteria}

The studies to be included should have been published in peer reviewed journals between (1 January 1990 and 11 November 2019); have a prospective design, with at least one baseline and one follow-up measure; including a parallel control group; allow for at least 3 months of follow-up; report the results in a way that could allow the calculations of risk ratios or equivalent association measures (e.g. odds ratios). Exclusion criteria were conference abstracts, book chapters or articles not reporting empirical results; studies based on selected samples, not representative of the underlying general populations (e.g. military personnel, patients with specific diagnoses, including psychiatric disorders). Studies reporting conflict of interest were not excluded, but information on conflict of interests presented in the included papers are presented in table S2. The study selection processes, and the articles excluded in each step are further outlined in figure 1. Details on excluded articles can be found in tables S3 and S4.

\section{Assessment of risk of bias}

The literature was evaluated using a priori established protocols and was reported according to the Preferred Reporting Items for Systematic Reviews and Meta-Analyses (PRISMA; www.prisma-statement. org/). The screening of the retrieved scientific articles was conducted using established protocols based on Cochrane's templates ROBINS-I and ROBINS-E. These templates have been developed by building upon tools for risk of bias assessment of randomised trials, diagnostic test accuracy studies and observational studies of interventions, and has been developed by members of the Cochrane Bias Methods Group and the Cochrane Non-Randomised Studies of Interventions Methods Group. Each article was initially reviewed by two of the authors. If the pair disagreed, a third reviewer among the listed authors resolved the conflict. None of the reviewers had a conflict of interest regarding the articles that they rated.

\section{TABLE 1 PICO (Population, Intervention, Comparison, and Outcome) schema}

\begin{tabular}{ll} 
Population & General population samples of nonsmokers, any age \\
Intervention/exposure & Self-reported current or ever use of e-cigarettes, with or without nicotine \\
Comparison group & Self-reported non-use of e-cigarettes throughout the study period \\
Outcome & Self-reported ever or current use of conventional cigarettes at follow-up \\
\hline
\end{tabular}




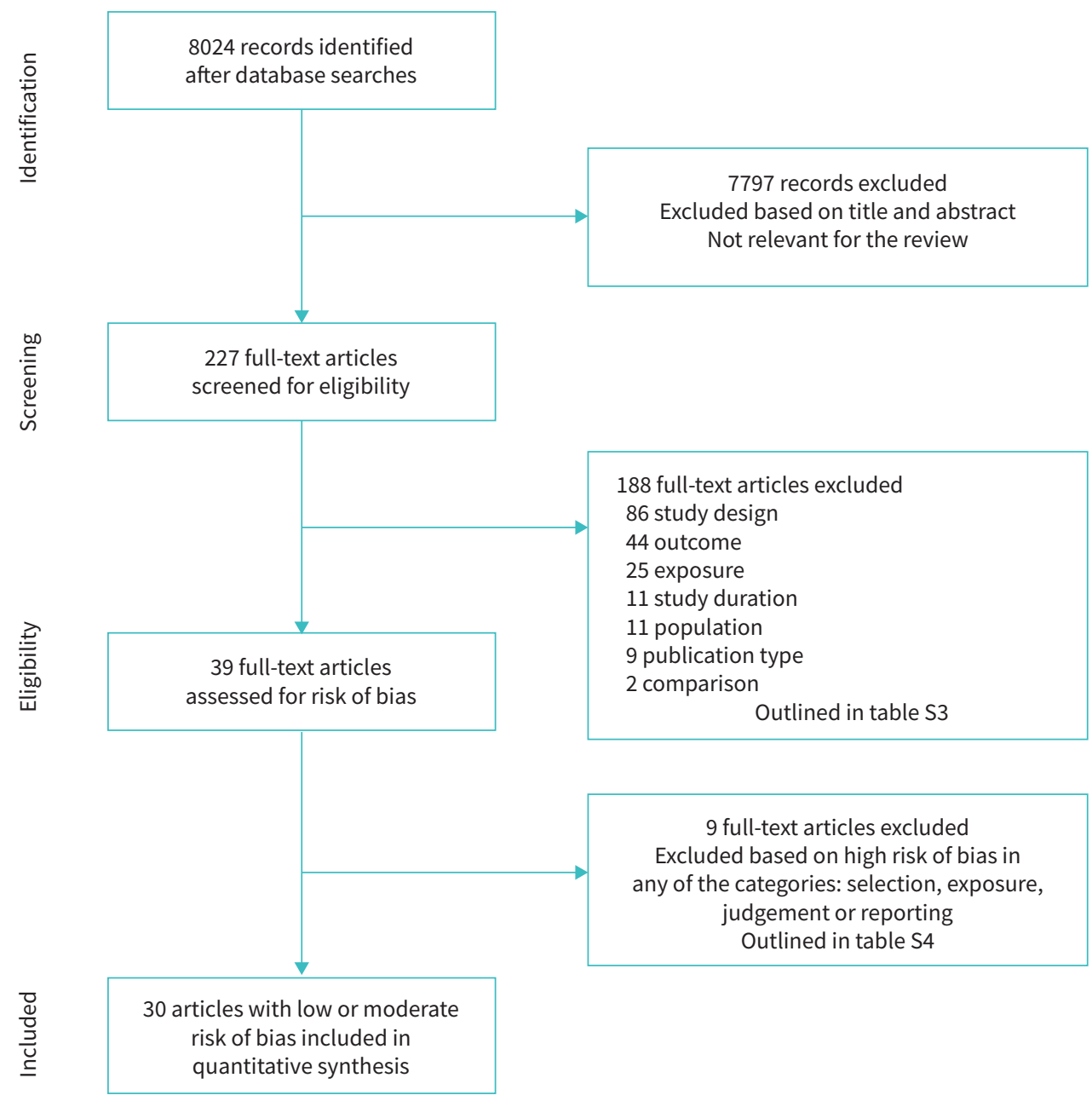

FIGURE 1 Study selection flowchart. Only longitudinal studies with at least three months study duration and judged to be at low or moderate risk of bias were included in the review.

Articles read in full text but judged not to be relevant to this review are listed in table S3. Articles that were considered relevant underwent a risk of bias assessment. Only prospective studies with a low or moderate risk of bias were included in the analysis. If an article was considered to have a high risk of bias in any of the assessed categories, selection, exposure, judgement or reporting, it was not included in the analysis (figure 1). Articles excluded due to high risk of bias are presented in table S4.

\section{Data extraction and statistical analysis}

The methodology of selected studies was examined, and main features were extracted and compiled including authors, date of publication, study design, subjects and sample size, main quantitative results and odds ratio values. For analysis, we have used data from studies where it has been possible to retrieve either absolute numbers or percentages for each group (unadjusted analysis), or where the study has presented the result as adjusted or unadjusted odds ratios. All analyses were conducted according to the $a$ priori analysis plan, with two main outcomes: ever-smoking and current smoking. Unadjusted data and adjusted data were analysed separately, and studies where odds ratios could not be calculated, were not included in the meta-analyses, but included narratively. However, no such studies were included in the review.

To assess the association between e-cigarette use and subsequent smoking, relevant data were combined in a random-effects meta-analysis, combined with inverse variance using Review Manager version 5.3 (the Nordic Cochrane Centre, the Cochrane Collaboration, Copenhagen, Denmark). We also considered the following subgroups: duration of follow-up ( $\leqslant 12$ months or $>12$ months), age ( $<18$ years old or $\geqslant 18$ years old, based on the legal age in many countries) and sex (male or female). Subgroups for further analyses 
were defined a priori. During the evaluation, several studies were shown to build on the same, or overlapping cohorts. In these cases, only the most complete dataset from the overlapping study populations was used in the meta-analysis. One study presented several sets of adjusted data, with different numbers of confounders. In that case the least adjusted model, which among others contained the variables sex, age and socioeconomic factors, was chosen to make the studies included in the analysis as comparable as possible.

A sensitivity analysis was performed comparing studies using different control groups (never-smokers versus nonsmokers; and never-e-cigarette users versus noncurrent e-cigarette users). As the majority of studies were conducted in North America, a sensitivity analysis excluding these studies was also performed. A putative publication bias was assessed using a funnel plot. No study was financially supported by companies associated with the tobacco industry or the e-cigarette industry.

\section{Grading of evidence}

We followed the GRADE guidelines to appraise the strength of evidence for the association resulting from the meta-analysis [20-23]. In this approach, direct evidence from longitudinal studies starts at high certainty $(++++)$ and is subsequently downrated based on risk of bias, indirectness, imprecision, and heterogeneity to levels of moderate $(+++)$, low $(++)$ and very low $(+)$ certainty of evidence. Both unadjusted and adjusted estimates were taken into account when grading the evidence.

Results

Description of the studies included in the review

30 peer-reviewed articles from 22 longitudinal studies were included in this review. 20 of these articles were published in the USA [10, 12, 13, 24-40], two in Canada [9, 14], one in Mexico [41] and seven in Europe [42-48]. The study populations included on average $48 \%$ females, varying from $42 \%$ [25] to $68 \%$ [33]. The majority of publications (21) were based on adolescents (i.e. younger than 19 years). In six studies the study population was older than 18 years [29, 33, 34, 37-39], while three studies included a population with a mixed age span [28, 35, 36]. Information regarding the education level was not explicitly expressed in the majority of studies. For studies involving adolescents, one study reported that $45 \%$ of the guardians had at least 4 years of college education [30], while two other studies report that $70 \%$ had more than 12 years education [25, 26]. For studies with a mixed population, the education level was reported to be at level 7 of 10 [35], or that $84 \%$ had an education greater than high school [28]. For studies involving adults, around $70 \%$ of the study population had some form of college education [34, 38].

Ten studies assembled data through questionnaires distributed during school hours [9, 12, 14, 24-26, 31, $32,40,42]$; seven studies used questionnaires that were distributed by mail or in other ways [10, 28, 41, 45-48]; five studies collected data through in-person interviews [27, 29, 30, 34, 36]; and five studies used web-based surveys [33, 38, 39, 43, 44]. The duration of the follow-up varied between 4 months and 2 years. 27 studies [9, 10, 12-14, 24-26, 28-31, 33-44, 46-48] emanate from never smoking individuals at baseline. The other three studies are based on noncurrent smokers [27, 32, 45] and may thus include former smokers even though they are based on adolescents.

\section{Incident smoking among e-cigarette users and non-users}

The exposure group is described as e-cigarette users in most studies, and as current e-cigarette users in three studies [9, 14, 27]. In one study the exposure group is referred to as electronic nicotine delivery system users [28], which may also include the use of other electronic noncombustible tobacco products. The control group is referred to as never e-cigarette users [10, 13, 24-26, 28, 30-37, 39-43, 45, 47], and as noncurrent e-cigarette users in six studies [9, 14, 27, 29, 44, 46].

Unadjusted data were presented in 17 of the included studies, including a total of 89076 participants. Among baseline never-smokers, the unadjusted OR for cigarette smoking initiation ranged between 2.39 and 12.31 across studies for those who had ever tried e-cigarettes compared with those who had never tried e-cigarettes (figure S1). Only one study presented unadjusted data for nonsmokers [32]. The OR was 6.70 favouring an association between smoking initiation and e-cigarette use. When combining the data in a random-effects meta-analysis, the pooled unadjusted OR for smoking initiation was 4.68 (95\% CI 3.646.02) for baseline e-cigarette users compared with baseline non-e-cigarette users (figure S1).

Adjusted data were presented in 20 studies (figure 2). The data were adjusted for sex (19 out of 20), age (15 out of 20) and socioeconomic factors (13 out of 20), but there was a large heterogeneity with respect to how these adjustments were made. The adjusted OR ranged between 1.36 and 11.37 for baseline never-smokers, and between 2.51 and 7.08 for noncurrent smokers (figure 2). The pooled adjusted OR for 


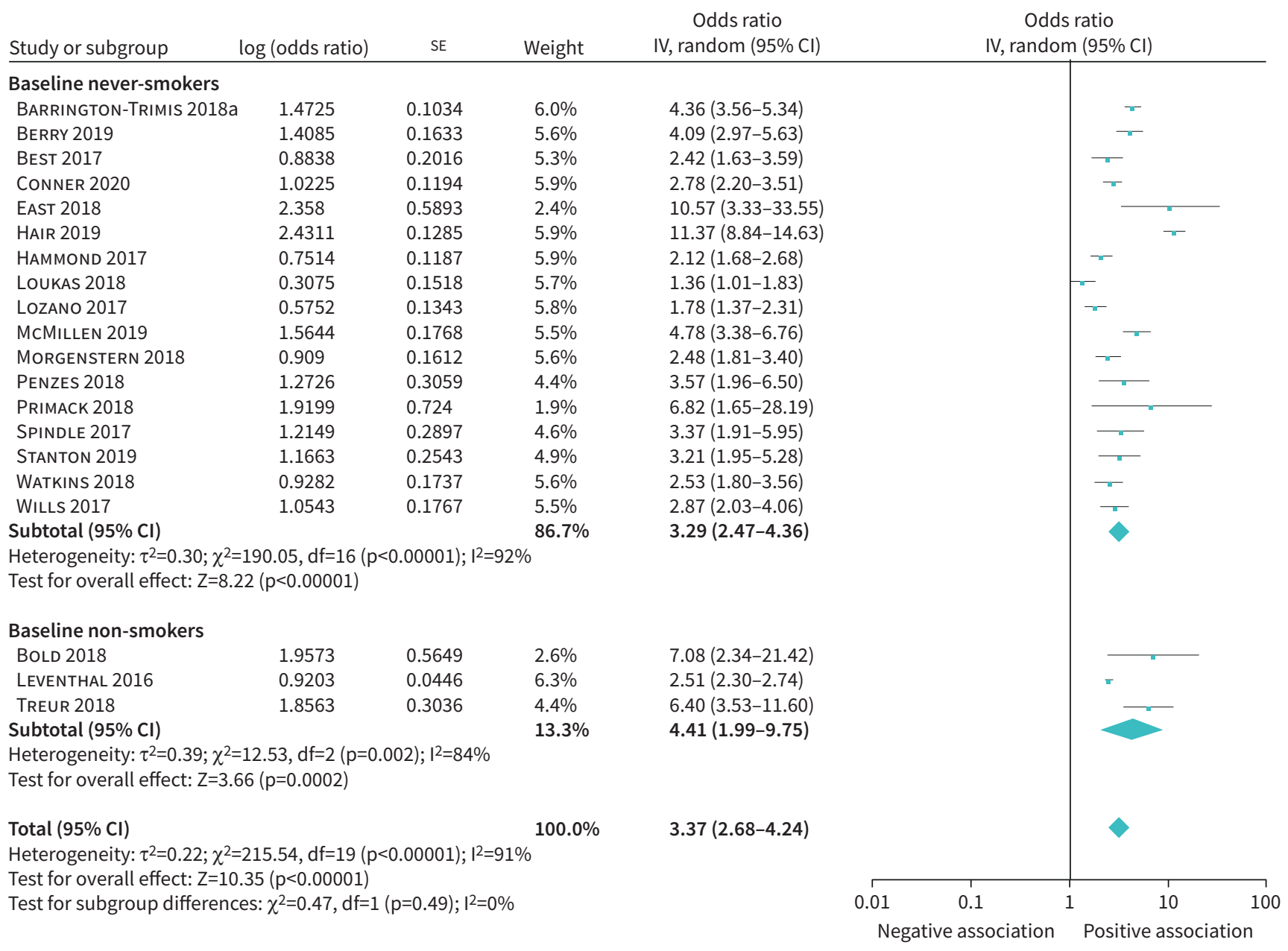

FIGURE 2 E-cigarette use and subsequent initiation or recurrence of cigarette smoking. Meta-analysis of adjusted odds of smoking initiation among e-cigarette users at baseline compared with non-e-cigarette users at baseline. Studies were adjusted for sex (19 out of 20 ), age (15 out of $20)$ and socioeconomic factors (13 out of 20 ).

smoking at follow-up among baseline e-cigarette users compared with baseline non-e-cigarette users was 3.37 (95\% CI 2.68-4.24) (figure 2).

Sensitivity analyses were performed to assess the robustness of the estimates by omitting: 1) studies with nonsmokers at baseline (figure S2a, b); 2) studies with noncurrent e-cigarette user as a control group (figure S3a, b); 3) studies with never e-cigarette users as a control group (figure S4a, b); and, 4) studies from the USA (figure S5a, b). All analyses were confirmatory of the overall findings. Stratified analyses of studies with a short ( $\leqslant 12$ months) or a long ( $>12$ months) follow-up (figure S6a, b), and of studies among adolescents ( $<18$ years old) or adults ( $\geqslant 18$ years old), showed similar results (figure S7a, b). The available data did not allow subgroup analyses by sex.

\section{Certainty of evidence}

The strength of the evidence concerning the association between e-cigarette use and smoking initiation was judged to be moderate $(+++)$ due to risk of bias in any of the categories: selection, exposure, judgement or reporting (table 2). At the same time, the material was extensive, and all studies supported a positive association albeit with large heterogeneity with regards to the strength of the outcome. For the subgroup adults aged 18 years or older, the number of studies was lower, and most included American college students, which can be considered a selected population. The strength of evidence for this subgroup was therefore downrated to low $(++)$ (table 2). 


\begin{tabular}{|c|c|c|c|c|c|c|}
\hline $\begin{array}{l}\text { Association with } \\
\text { smoking initiation }\end{array}$ & Participants & $\begin{array}{l}\text { Number of } \\
\text { studies }\end{array}$ & $\begin{array}{l}\text { Risk difference } \\
\qquad(95 \% \mathrm{Cl})^{\#}\end{array}$ & OR $(95 \% \mathrm{Cl})$ & $\begin{array}{l}\text { Certainty of } \\
\text { evidence }\end{array}$ & Down rating \\
\hline Full material & 89076 & $\begin{array}{l}\text { Unadjusted: } 17 \\
\text { Adjusted: } 20\end{array}$ & $0.22(0.14-0.28)$ & $\begin{array}{c}\text { Unadjusted OR: } 4.68 \\
(3.64-6.02) \\
\text { Adjusted OR: } 3.37 \\
(2.68-4.24)\end{array}$ & Moderate $(+++)$ & -1 risk of bias \\
\hline $\begin{array}{l}\text { Short-term follow-up } \\
\text { ( } \leqslant 12 \text { months) }\end{array}$ & 64016 & $\begin{array}{l}\text { Unadjusted:13 } \\
\text { Adjusted: } 18\end{array}$ & $0.18(0.14-0.23)$ & $\begin{array}{c}\text { Unadjusted OR: } 4.60 \\
\text { (4.01-5.29) } \\
\text { Adjusted OR: } 4.13 \\
(3.16-5.40)\end{array}$ & Moderate $(+++)$ & -1 risk of bias \\
\hline $\begin{array}{l}\text { Long-term follow-up } \\
\text { (>12 months) }\end{array}$ & 31265 & $\begin{array}{l}\text { Unadjusted: } 8 \\
\text { Adjusted: } 6\end{array}$ & $0.25(0.13-0.38)$ & $\begin{array}{c}\text { Unadjusted OR: } 4.71 \\
(3.03-7.34) \\
\text { Adjusted OR: } 2.84 \\
(1.85-4.36)\end{array}$ & Moderate $(+++)$ & -1 risk of bias" \\
\hline$<18$ years & 761399 & $\begin{array}{l}\text { Unadjusted: } 13 \\
\text { Adjusted: } 15\end{array}$ & $0.07(0.04-0.10)$ & $\begin{array}{c}\text { Unadjusted OR: } 5.11 \\
\text { (3.89-6.72) } \\
\text { Adjusted OR: } 2.94 \\
\text { (2.49-3.48) }\end{array}$ & Moderate $(+++)$ & -1 risk of bias \\
\hline$\geqslant 18$ years & 18438 & $\begin{array}{l}\text { Unadjusted: } 4 \\
\text { Adjusted: } 4\end{array}$ & $0.16(0.11-0.22)$ & $\begin{array}{c}\text { Unadjusted OR: } 3.04 \\
(2.57-3.58) \\
\text { Adjusted OR: } 3.19\end{array}$ & Low (++) & $\begin{array}{c}-1 \text { risk of bias } \\
-1 \text { material with several } \\
\text { limitations }{ }^{+, \S}\end{array}$ \\
\hline
\end{tabular}

(1.44-7.05)

\#: calculated from unadjusted values; ๆ: material with several deficits and limitations; ${ }^{+}$: indirectness, three out of five studies are performed on college students in the USA; ${ }^{\S}$ : the analysis is based on a limited number of studies.

\section{Current smoking among e-cigarette users and non-users}

13 publications based on 10 cohorts [10, 12, 24, 25, 28-30, 34, 38, 39, 41, 47, 48] assessed the association between e-cigarette use and subsequent current smoking. Current smoking was in most studies defined as smoking at least once over the past 30 days and may thus include both daily smokers as well as less-regular smokers. Most studies presented a dichotomous 'yes' or 'no' outcome in the analysis, and a sensitivity analysis could thus not be performed based on smoking frequency. All studies were based on baseline never-smokers, and the exposure group was e-cigarette users. The control group was never-e-cigarette users in all studies except one, which was based on non-e-cigarette users [29]. The follow-up period varied between 6 and 24 months.

Seven studies, with a total of 39086 participants, present unadjusted values in form of number or percentage of the participants reporting current smoking [10, 25, 29, 30, 39, 41, 48]. Among baseline never-smokers, the unadjusted OR for current cigarette smoking at follow-up ranged between 2.16 and 6.13 across studies for those who had used e-cigarettes at baseline compared with those who were non-e-cigarette users (figure S8). When combining the data in a random-effects meta-analysis, the pooled unadjusted OR for smoking initiation was 3.51 (95\% CI 2.87-4.29) for baseline e-cigarette users compared with baseline non-e-cigarette users (figure S8).

Nine studies present adjusted data $[10,25,28,30,34,38,39,41,48]$. The choice and number of covariates differed between the studies. Eight out of nine adjusted for sex and age, and five out of nine adjusted for socioeconomic status. There is a large variability in how these adjustments have been made. The adjusted OR ranged between 1.47 and 17.33 for baseline never-smokers (figure 3). The pooled adjusted OR for current smoking among baseline e-cigarette users compared with baseline non-e-cigarette users was 3.89 (95\% CI 2.16-7.00) (figure 3). Stratified analysis of studies with a short ( $\leqslant 12$ months) or a long ( $>12$ months) follow-up (figure S9), and studies assessing transitions among adolescents ( $<18$ years old) or adults ( $\geqslant 18$ years old) showed the same association (figure S10). Stratified data by sex were not available.

\section{Certainty of evidence}

Due to risk of bias in any of the categories selection, exposure, judgement or reporting, the certainty of the evidence for an association between e-cigarette use and subsequent smoking for the whole material, and for the material entailing adolescents below 18 years old was considered to be moderate $(+++)$. Despite a significant heterogeneity between studies with respect to association estimates, all studies supported an 


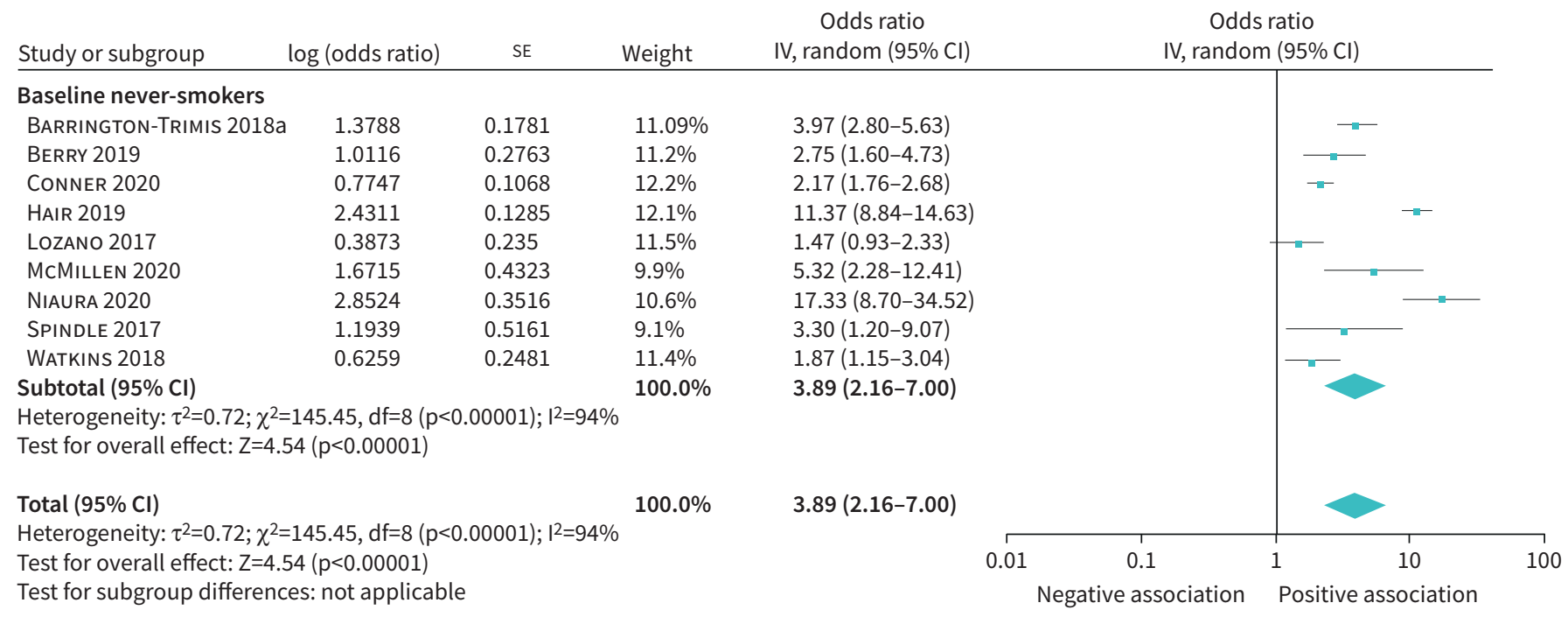

FIGURE 3 E-cigarette use and self-reported current smoking. Meta-analysis of adjusted odds of current smoking among e-cigarette users at baseline compared with non-e-cigarette users at baseline. Studies were adjusted for sex (8 out of 9), and socioeconomic factors (5 out of 9).

association between e-cigarette use at baseline and current smoking at follow-up. For the subgroup with longer follow-up the certainty of evidence was considered to be low $(++)$, and very low for the subgroup of adults ( $\geqslant 18$ years of age) $(+)$. Deductions were connected to general limitations in the included studies, and to the limited number of studies (table S5).

\section{Assessment of publication bias}

Funnel plots did not indicate any publications bias in the material (figures S11 and S12).

\section{Discussion}

This systematic review and meta-analysis, based on 30 studies, supports an association between e-cigarette use and subsequent smoking, with moderate strength of evidence. E-cigarette users showed an increased probability of future cigarette smoking even after adjusting for potential confounders, suggesting that e-cigarette use by itself could be considered a predictor of subsequent conventional smoking. The certainty of evidence, which was rated using GRADE, was estimated to be moderate for the full material. A subgroup analyses based on the age of study participants supported a stronger association between e-cigarette use and smoking among adolescents. These results are in agreement with other systematic reviews on the subject, particularly those including longitudinal studies [16-19]. Due to the rapid increase in published studies, the material included in this review and meta-analysis is more extensive than in the aforementioned reviews, thereby further supporting the association between e-cigarette use and an increased risk of subsequent smoking initiation or relapse among nonsmokers.

The associations presented in this meta-analysis are not necessarily causal. The likelihood for specific individuals to initiate smoking, as well as e-cigarette use, may depend on multiple factors including genetics, attitude towards smoking, harm perception, social norms, and mental health issues [49, 50]. If a longitudinal association between two behaviours is independent of the order of onset, there is an increased likelihood that shared risk factors may explain the association. In fact, several studies reported a bidirectional association between e-cigarettes and other tobacco products [51]. E-cigarette use appears to be followed by an increased probability of subsequent smoking, but there was also an increased probability of e-cigarette initiation among current smokers [44, 52]. A similar bidirectional association has also been found between use of other tobacco products, such as snus [53-55]. It is thus possible that a third factor, such as the willingness to experiment with addictive substances, or factors increasing the risk of developing nicotine addiction, contributes to both behaviours. The onset of the behaviour may then be related to chance or unmeasured confounders $[19,56]$. However, it should be noted that several studies presented a transition from e-cigarette use to smoking especially among low-risk youths, suggesting that e-cigarette use may not simply be a marker of liability to smoking initiation [10, 12, 14, 35]. Furthermore, the association between e-cigarette use and smoking initiation remained after adjusting for confounding factors, indicating robustness of the observed associations. 
Even if the included studies all showed an increased risk of smoking initiation among nonsmoking e-cigarette users when compared to non-e-cigarette users, the data presented were heterogenous. One factor putatively contributing to the heterogeneity could be the follow-up time, which varied from 4 months to up to 2 years. Shorter follow-up times may putatively be more trustworthy in capturing events associated with baseline measures. The dropout rate may also be higher when employing a longer follow-up period. However, subgroup analysis separating the studies with regards to the time to follow-up still demonstrated similar results, with the quality of the estimate being rated as moderate.

Age has been shown to be an important risk factor when assessing the transition from initiation to current smoking [57-59]. The age at baseline of the study participants in this review varied between 11 [43, 48] and 34 years [38], which might also contribute to the heterogeneity between studies. In fact, misclassification of events typically occurring in adolescence (e.g. first smoking episodes) is common when recalled several years later, both due to memory failure and to recanting [60]. Thus, in studies conducted among adults some self-reported never-smokers may have already initiated smoking, albeit not progressing to regular smoking. This misclassification may have biased the estimates of association with e-cigarette use in any direction. In fact, even if the results obtained with stratified analyses of two age groups ( $<18$ versus $\geqslant 18$ years) were consistent, the quality of the estimations was higher for studies assessing smoking initiation and current use among adolescents. However, the fact that e-cigarette use is prospectively associated with smoking even among adults, further suggests a role of e-cigarette use as promoter of cigarette smoking.

In this review we separated studies assessing ever-smoking and current smoking at follow-up. As current use often corresponds to more established smoking, these studies may more accurately reflect the transition from e-cigarettes to combustible cigarettes. However, there was no significant difference in the associations outcome when comparing these two subcategories of outcomes. This similarity might be due to the fact that current smoking in most studies was defined as past 30 days' use and may thus include both sporadic and daily smoking because the outcome was treated as dichotomous in the analysis. It was thus not possible to perform a sensitivity analysis to outline the impact by e-cigarette use on daily versus less-than-daily smoking.

Regulations regarding advertisement and nicotine content of e-cigarettes vary extensively between countries [61-63]. In the USA, the allowed nicotine concentration in the e-liquid is higher than in the European Union. If nicotine content affects the likelihood of smoking initiation [64], the strength of the associations might be expected to vary between countries; however, a sensitivity analysis excluding studies from the USA yielded similar associations between e-cigarette use and increased risk of smoking initiation as in the whole material.

\section{Limitations and strengths}

The major strength of this review is that it is based on 30 studies from different countries using a longitudinal prospective design. Furthermore, to avoid inclusion of articles that were subject to major limitations the review conducted here only included prospective studies with a low or moderate risk of bias. Cross-sectional studies, or longitudinal studies with a shorter duration than 3 months were also not included. Another strength of this manuscript is that it not only examines the association between e-cigarette use and smoking initiation but also recurrence to smoking.

Several limitations should be mentioned. Most studies did not assess the type of e-cigarette device used, the frequency of use, the composition of e-liquid or whether e-cigarettes contained nicotine or not. The newer models of e-cigarettes allow exposure of higher nicotine levels as compared to first-generation e-cigarettes, and the composition of the e-liquid used, and how it is vaped, may influence blood levels of nicotine [65-67]. This information is especially important since nicotine exposure may be fundamental for subsequent smoking behaviour [64]. However, it should be noted that the assessment of these features of nicotine exposure is impaired by the fact that many e-cigarette users are unaware of the content of nicotine in the e-cigarettes they are using, and even the exact type of electronic device may not be clear [62, 68, 69]. The frequency of use of cigarette smoking is generally not investigated, therefore the distinction between regular and experimental use is seldom possible. Furthermore, current use is in most studies defined as any use of cigarettes in the past 30 days, and may thus not represent regular smoking.

The adjustment for potential confounding factors was very different across studies. It was thus not possible to grade the effect size of the association. Grading of evidence thereby relates to the certainty of evidence for an association between e-cigarette use and smoking initiation or recurrence. The size of the exposed populations (e-cigarette users) was generally low, and the dropout rate was relatively high, therefore 
limiting the statistical power and increasing the potential for selection bias. In this review we did not exclude articles due solely to their attrition, but this aspect was considered in the judgement of the risk of bias and in the grading of evidence.

\section{Conclusions}

This review and meta-analysis suggests that e-cigarette use may predict the initiation or recurrence of cigarette smoking. E-cigarette users had an increased probability of future cigarette smoking even after adjusting for potential confounders, suggesting that e-cigarette use by itself could be considered a predictor of subsequent conventional smoking.

Acknowledgements: We greatly acknowledge the assistance provided by Martin Norman, Elisabeth Gustafsson, Hanna Olofsson and Karin Stenström at the Swedish Agency for Health Technology Assessment and Assessment of Social Services (SBU), Stockholm, Sweden.

Author contributions: This project was initiated in collaboration between The Public Health Agency of Sweden and the SBU. All authors followed the procedures described by PRISMA when assessing studies. CR performed statistical analysis. L. Adermark drafted the manuscript, which was revised and edited by all coauthors.

Conflict of interest: L. Adermark reports grants from Swedish Research Council during the conduct of the study. M.R. Galanti has nothing to disclose. C. Ryk has nothing to disclose. H. Gilljam has nothing to disclose. L. Hedman has nothing to disclose.

Support statement: The project was under governmental support. The first author was funded by the Swedish Research Council (grant 2018-0258). Funding information for this article has been deposited with the Crossref Funder Registry.

\section{References}

1 King BA, Patel R, Nguyen KH, et al. Trends in awareness and use of electronic cigarettes among US adults, 2010-2013. Nicotine Tob Res 2015; 17: 219-227.

2 Brozek GM, Jankowski M, Lawson JA, et al. The prevalence of cigarette and e-cigarette smoking among students in Central and Eastern Europe-results of the YUPESS study. Int J Environ Res Public Health 2019; 16: 2297.

3 Cullen KA, Gentzke AS, Sawdey MD, et al. e-Cigarette use among youth in the United States, 2019. JAMA 2019; 322: 2095-2103.

4 Farsalinos K, Barbouni A, Niaura R. Changes from 2017 to 2018 in e-cigarette use and in ever marijuana use with e-cigarettes among US adolescents: analysis of the National Youth Tobacco Survey. Addiction 2020; 116: 139-149.

5 Gentzke AS, Creamer M, Cullen KA, et al. Vital signs: tobacco product use among middle and high school students - United States, 2011-2018. MMWR Morb Mortal Wkly Rep 2019; 68: 157-164.

6 Bauld L, MacKintosh AM, Eastwood B, et al. Young people's use of e-cigarettes across the United Kingdom: findings from five surveys 2015-2017. Int J Environ Res Public Health 2017; 14: 973.

7 Hedman L, Backman H, Stridsman C, et al. Association of electronic cigarette use with smoking habits, demographic factors, and respiratory symptoms. JAMA Netw Open 2018; 1: e180789.

8 Hammond D, Reid JL, Rynard VL, et al. Prevalence of vaping and smoking among adolescents in Canada, England, and the United States: repeat national cross sectional surveys. BMJ 2019; 365: 12219.

9 Hammond D, Reid JL, Cole AG, et al. Electronic cigarette use and smoking initiation among youth: a longitudinal cohort study. CMAJ 2017; 189: E1328-E1336.

10 Berry KM, Fetterman JL, Benjamin EJ, et al. Association of electronic cigarette use with subsequent initiation of tobacco cigarettes in US youths. JAMA Netw Open 2019; 2: e187794.

11 Jongenelis MI, Jardine E, Kameron C, et al. E-cigarette use is associated with susceptibility to tobacco use among Australian young adults. Int J Drug Policy 2019; 74: 266-273.

12 Barrington-Trimis JL, Urman R, Berhane K, et al. E-cigarettes and future cigarette use. Pediatrics 2016; 138 : e20160379.

13 Wills TA, Sargent JD, Gibbons FX, et al. E-cigarette use is differentially related to smoking onset among lower risk adolescents. Tob Control 2016; 26: 534-539.

14 Aleyan S, Cole A, Qian W, et al. Risky business: a longitudinal study examining cigarette smoking initiation among susceptible and non-susceptible e-cigarette users in Canada. BMJ Open 2018; 8: e021080.

15 Levy DT, Warner KE, Cummings KM, et al. Examining the relationship of vaping to smoking initiation among US youth and young adults: a reality check. Tob Control 2019; 28: 629-635. 
16 Glasser A, Abudayyeh H, Cantrell J, et al. Patterns of E-cigarette use among youth and young adults: review of the impact of e-cigarettes on cigarette smoking. Nicotine Tob Res 2019; 21: 1320-1330.

17 Khouja JN, Suddell SF, Peters SE, et al. Is e-cigarette use in non-smoking young adults associated with later smoking? A systematic review and meta-analysis. Tob Control 2020; 30: 8-15.

18 Soneji S, Barrington-Trimis JL, Wills TA, et al. Association between initial use of e-cigarettes and subsequent cigarette smoking among adolescents and young adults: a systematic review and meta-analysis. JAMA Pediatr 2017; 171: 788-797.

19 Chan GCK, Stjepanovic D, Lim C, et al. Gateway or common liability? A systematic review and meta-analysis of studies of adolescent e-cigarette use and future smoking initiation. Addiction 2020; 116: 743-756.

20 Atkins D, Best D, Briss PA, et al. Grading quality of evidence and strength of recommendations. BMJ 2004; 328: 1490.

21 Guyatt GH, Oxman AD, Vist GE, et al. GRADE: an emerging consensus on rating quality of evidence and strength of recommendations. BMJ 2008; 336: 924-926.

22 Morgan RL, Thayer KA, Santesso N, et al. A risk of bias instrument for non-randomized studies of exposures: a users' guide to its application in the context of GRADE. Environ Int 2019; 122: 168-184.

23 Schunemann HJ, Cuello C, Akl EA, et al. GRADE guidelines: 18. How ROBINS-I and other tools to assess risk of bias in nonrandomized studies should be used to rate the certainty of a body of evidence. $J$ Clin Epidemiol 2019; 111: 105-114.

24 Barrington-Trimis JL, Bello MS, Liu F, et al. Ethnic differences in patterns of cigarette and e-cigarette use over time among adolescents. J Adolesc Health 2019; 65: 359-365.

25 Barrington-Trimis JL, Kong G, Leventhal AM, et al. E-cigarette use and subsequent smoking frequency among adolescents. Pediatrics 2018; 142: e20180486.

26 Barrington-Trimis JL, Leventhal AM, Alonzo TA, et al. Performance of cigarette susceptibility index among e-cigarette and hookah users. Drug Alcohol Depend 2018; 183: 43-50.

27 Bold KW, Kong G, Camenga DR, et al. Trajectories of e-cigarette and conventional cigarette use among youth. Pediatrics 2018; 141: 20171832.

28 Hair EC, Romberg AR, Niaura R, et al. Longitudinal tobacco use transitions among adolescents and young adults: 2014-2016. Nicotine Tob Res 2019; 21: 458-468.

29 Kasza KA, Borek N, Conway KP, et al. Transitions in tobacco product use by US adults between 2013-2014 and 2014-2015: findings from the PATH study Wave 1 and Wave 2. Int J Environ Res Public Health 2018; 15 : 2515.

30 Watkins SL, Glantz SA, Chaffee BW. Association of noncigarette tobacco product use with future cigarette smoking among youth in the Population Assessment of Tobacco and Health (PATH) study, 2013-2015. JAMA Pediatr 2018; 172: 181-187.

31 Leventhal AM, Strong DR, Kirkpatrick MG, et al. Association of electronic cigarette use with initiation of combustible tobacco product smoking in early adolescence. JAMA 2015; 314: 700-707.

32 Leventhal AM, Stone MD, Andrabi N, et al. Association of e-cigarette vaping and progression to heavier patterns of cigarette smoking. JAMA 2016; 316: 1918-1920.

33 Loukas $\mathrm{A}$, Marti $\mathrm{CN}$, Cooper $\mathrm{M}$, et al. Exclusive e-cigarette use predicts cigarette initiation among college students. Addict Behav 2018; 76: 343-347.

34 McMillen R, Klein JD, Wilson K, et al. E-cigarette use and future cigarette initiation among never smokers and relapse among former smokers in the PATH study. Public Health Rep 2019; 134: 528-536.

35 Primack BA, Soneji S, Stoolmiller M, et al. Progression to traditional cigarette smoking after electronic cigarette use among US adolescents and young adults. JAMA Pediatr 2015; 169: 1018-1023.

36 Stanton CA, Bansal-Travers M, Johnson AL, et al. Longitudinal e-cigarette and cigarette use among US youth in the PATH study (2013-2015). J Natl Cancer Inst 2019; 111: 1088-1096.

37 Primack BA, Shensa A, Sidani JE, et al. Initiation of traditional cigarette smoking after electronic cigarette use among tobacco-naive US young adults. Am J Med 2018; 131: 443.e441-443.e449.

38 Niaura R, Rich I, Johnson AL, et al. Young adult tobacco and e-cigarette use transitions: examining stability using multistate modeling. Nicotine Tob Res 2020; 22: 647-654.

39 Spindle TR, Hiler MM, Cooke ME, et al. Electronic cigarette use and uptake of cigarette smoking: A longitudinal examination of U.S. college students. Addict Behav 2017; 67: 66-72.

40 Wills TA, Knight R, Sargent JD, et al. Longitudinal study of e-cigarette use and onset of cigarette smoking among high school students in Hawaii. Tob Control 2017; 26: 34-39.

41 Lozano P, Barrientos-Gutierrez I, Arillo-Santillan E, et al. A longitudinal study of electronic cigarette use and onset of conventional cigarette smoking and marijuana use among Mexican adolescents. Drug Alcohol Depend 2017; 180: 427-430.

42 Best C, Haseen F, Currie D, et al. Relationship between trying an electronic cigarette and subsequent cigarette experimentation in Scottish adolescents: a cohort study. Tob Control 2017; 27: 373-378.

43 East K, Hitchman SC, Bakolis I, et al. The association between smoking and electronic cigarette use in a cohort of young people. J Adolesc Health 2018; 62: 539-547. 
323-334.

46 Morgenstern M, Nies A, Goecke M, et al. E-cigarettes and the use of conventional cigarettes. Dtsch Arztebl Int 2018; 115: 243-248.

47 Conner M, Grogan S, Simms-Ellis R, et al. Do electronic cigarettes increase cigarette smoking in UK adolescents? Evidence from a 12-month prospective study. Tob Control 2017; 27: 365-372.

48 Conner M, Grogan S, Simms-Ellis R, et al. Evidence that an intervention weakens the relationship between adolescent electronic cigarette use and tobacco smoking: a 24-month prospective study. Tob Control 2020; 29: 425-431.

49 Allegrini AG, Verweij KJH, Abdellaoui A, et al. Genetic vulnerability for smoking and cannabis use: associations with e-cigarette and water pipe use. Nicotine Tob Res 2019; 21: 723-730.

50 Treur JL, Demontis D, Smith GD, et al. Investigating causality between liability to ADHD and substance use, and liability to substance use and ADHD risk, using Mendelian randomization. Addict Biol 2019: 26; e12849.

51 Chaffee BW, Cheng J. Tobacco product initiation is correlated with cross-product changes in tobacco harm perception and susceptibility: Iongitudinal analysis of the Population Assessment of Tobacco and Health youth cohort. Prev Med 2018; 114: 72-78.

52 Vardavas $\mathrm{Cl}$, Filippidis FT, Agaku IT. Determinants and prevalence of e-cigarette use throughout the European Union: a secondary analysis of 26566 youth and adults from 27 Countries. Tob Control 2015; 24: 442-448.

53 Galanti MR, Rosendahl I, Post A, et al. Early gender differences in adolescent tobacco use: the experience of a Swedish cohort. Scand J Public Health 2001; 29: 314-317.

54 Haukkala A, Vartiainen E, de Vries H. Progression of oral snuff use among Finnish 13-16-year-old students and its relation to smoking behaviour. Addiction 2006; 101: 581-589.

55 Araneda D, Korhonen T, Laatikainen T, et al. Association of snus experimentation in late adolescence with daily cigarette smoking in early adulthood: a longitudinal study among Finnish men. Scand J Public Health 2020; 48: 638-645.

56 Cahn Z, Drope J, Douglas CE, et al. Applying the population health standard to the regulation of electronic nicotine delivery systems. Nicotine Tob Res 2020: 23; 780-789.

57 Chen J, Millar WJ. Age of smoking initiation: implications for quitting. Health Rep 1998; 9: 39-46.

58 Chaim $\mathrm{CH}$, Siu ER, Carvalho CFC, et al. Experimentation with tobacco during adolescence as a factor influencing treatment of smoking in adulthood. A retrospective cohort. Sao Paulo Med J 2019; 137: 234-240.

59 Huggett $\mathrm{SB}$, Keyes $\mathrm{M}$, lacono WG, et al. Age of initiation and transition times to tobacco dependence: early onset and rapid escalated use increase risk for dependence severity. Drug Alcohol Depend 2019; 202: 104-110.

60 Neugebauer R, Ng S. Differential recall as a source of bias in epidemiologic research. J Clin Epidemiol 1990; 43: 1337-1341.

61 Barrington-Trimis JL, Berhane K, Unger JB, et al. The e-cigarette social environment, e-cigarette use, and susceptibility to cigarette smoking. J Adolesc Health 2016; 59: 75-80.

62 Morean ME, Bold KW, Kong G, et al. Adolescents' awareness of the nicotine strength and e-cigarette status of JUUL e-cigarettes. Drug Alcohol Depend 2019; 204: 107512.

63 Pike JR, Tan N, Miller S, et al. The effect of e-cigarette commercials on youth smoking: a prospective study. Am J Health Behav 2019; 43: 1103-1118.

64 Kinnunen JM, Ollila $\mathrm{H}$, Minkkinen J, et al. Nicotine matters in predicting subsequent smoking after e-cigarette experimentation: a longitudinal study among Finnish adolescents. Drug Alcohol Depend 2019; 201: 182-187.

65 DeVito EE, Buta E, Sofuoglu M. E-cigarette nicotine dose and flavor: relationship with appeal, choice, and tobacco use amongst veterans with comorbid psychiatric disorders. Addict Behav 2019; 92: 53-57.

66 Voos N, Smith D, Kaiser L, et al. Effect of e-cigarette flavors on nicotine delivery and puffing topography: results from a randomized clinical trial of daily smokers. Psychopharmacology (Berl) 2020; 237: 491-502.

67 DeVito EE, Krishnan-Sarin S. E-cigarettes: impact of e-liquid components and device characteristics on nicotine exposure. Curr Neuropharmacol 2018; 16: 438-459.

68 Morean ME, Kong G, Cavallo DA, et al. Nicotine concentration of e-cigarettes used by adolescents. Drug Alcohol Depend 2016; 167: 224-227.

69 Sanders-Jackson AN, Tan AS, Bigman CA, et al. Knowledge about e-cigarette constituents and regulation: results from a national survey of US young adults. Nicotine Tob Res 2015; 17: 1247-1254. 